\title{
Measuring strain during a cylindrical grinding process using embedded sensors in a workpiece
}

\author{
Mridusmita Sarma $^{1}$, Florian Borchers ${ }^{2}$, Gerrit Dumstorff ${ }^{1}$, Carsten Heinzel ${ }^{2}$, and Walter Lang ${ }^{1}$ \\ ${ }^{1}$ Institute for Microsensors Actuators and Systems (IMSAS), University of Bremen, 28359, Bremen, Germany \\ ${ }^{2}$ Foundation Institute for Materials Science (IWT) and MAPEX Centre for Materials and Processes, \\ University of Bremen, Bremen 28359, Germany \\ Correspondence to: Mridusmita Sarma (msarma@imsas.uni-bremen.de)
}

Received: 6 June 2017 - Revised: 5 August 2017 - Accepted: 8 August 2017 - Published: 15 September 2017

\begin{abstract}
This paper presents the results of using a sensor-integrated workpiece for in situ measurement of strain during an outer-diameter cylindrical grinding process. The motivation of this work is to measure in situ process parameters using integrated sensors in a workpiece in order to characterize the manufacturing process. Resistive sensors that operate on the same principle as conventional strain gauges were fabricated on wafers made of steel using standard microtechnology and later the wafers were diced to form unique sensor-integrated steel components (sensor inlays). These inlays are embedded into a groove on the top surface of a cylindrical workpiece using epoxy adhesive. The workpiece is also made of the same steel as the sensor wafers and has similar properties due to a heat treatment process, thereby maintaining the homogeneity of the material over the whole contact area. The sensor-integrated workpiece was used to perform experiments in a Studer S41 highperformance cylindrical grinding machine. The sensor response to the internal strain was recorded during every grinding step starting from a depth of $1 \mathrm{~mm}$ down to $2 \mathrm{~mm}$ from the top surface. Such an application of sensor integration in materials for in situ process monitoring can be used in other manufacturing processes as well and this can help to observe internal loads (mechanical or thermal) in manufacturing processes.
\end{abstract}

\section{Introduction}

The motivation of this work is to produce a sensor-integrated workpiece for in situ measurement of process parameters like strain and temperature during manufacturing processes. This can be achieved by embedding a sensor-integrated component (referred to as the sensor inlay hereafter) into a standard workpiece used in manufacturing processes like grinding. By integrating sensors on the workpiece, a sensorial workpiece can be created which can be used to measure in situ strain and temperature in real time by acquiring the measured data from the integrated sensors during machining. The sensor inlay and the workpiece are made up of the same material. This ensures more homogeneity and is therefore more advantageous than integrating commercial strain sensors on the workpiece. In this way, the measurement uncertainties due to a so-called "wounding" effect caused by sensor embedding can be reduced to a large extent. This is one of the major advantages of using material-integrated sensors as opposed to using commercially available sensors which are externally mounted. Also, this is one of the most effective ways of measuring the process-induced strain and temperature in the workpiece as the forces on the material are directly translated into the underlying sensor layer. Also, the sensor inlay is universal and can be used in any manufacturing process and integrated into any workpiece made of the same material. The primary goal behind producing such a sensor-integrated workpiece is to characterize various manufacturing processes. By measuring strain and temperature in situ during any manufacturing process, it may be possible to correlate the internal loads that are specific to each manufacturing process and the corresponding material modifications induced by the various internal loads like stress, strain, temperature, etc., in the machined workpiece. This concept is termed process signatures of manufacturing processes (Brinksmeier et al., 2014). In this concept, it is assumed that all manufacturing processes 


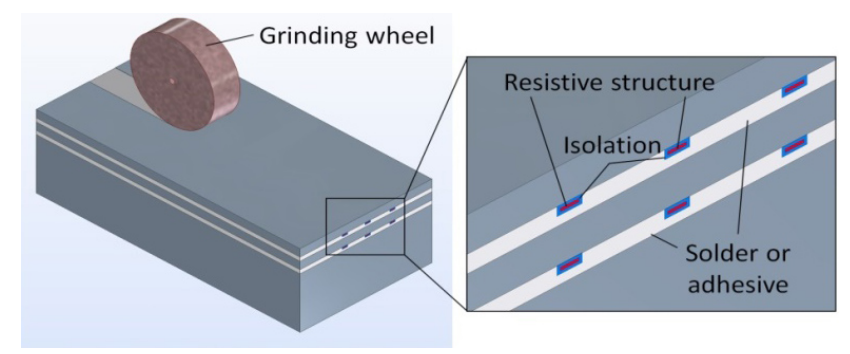

Figure 1. Schematic illustration of in situ measurement with a sensor-integrated workpiece in a surface grinding process.

fundamentally cause specific combinations of internal loads which can be broadly classified into three major types: thermal, mechanical, and chemical. Each manufacturing process contains at least one or a combination of these internal material loads. Hence, measuring the process parameters like external forces will help in identifying the specific internal loads of each manufacturing process. The measured data obtained from the integrated sensors are used to feed analytical models to calculate the internal loads implied by the process. This can lead to generation of transfer functions which can be used to design specific processes or process chains through the targeted desired surface properties independently of the manufacturing technologies. This procedure is so-called inverse process design. The schematic of the concept of using a sensor-integrated workpiece in a grinding process is shown in Fig. 1.

The application of a sensor-integrated workpiece for measuring in situ parameters in manufacturing processes is a novel concept of sensor integration in materials. Previously thin-film thermocouples were embedded in nickel substrates by electroplating for testing a welding process (Cheng et al., 2006). Strain gauges and thermocouples were together embedded in stainless steel substrates through the thin-film deposition technique, and it was tested in a milling process (Cheng et al., 2008; Choi et al., 2006). The use of a sensory workpiece for process monitoring has been reported earlier (Denkena et al., 2016) where commercial strain gauges were integrated into the workpiece. In this paper, strain sensors are fabricated directly on the workpiece material. The material used in this work for sensor fabrication is the high-grade heat treatable steel 42CrMo4 (AISI4140), which is a common material used in manufacturing processes. But this material is very prone to oxidation at room temperature and hence difficult to use as a substrate for microfabrication. Sensor fabrication on stainless steel substrates (Cheng et al., 2008) and 17-4PH steel substrates (Boedecker et al., 2011) has been reported, but fabrication on a reactive steel substrate like this is not yet state-of-the-art.

Two primary steps are involved in creating the sensorintegrated workpiece as mentioned above (Dumstorff et al., 2016).

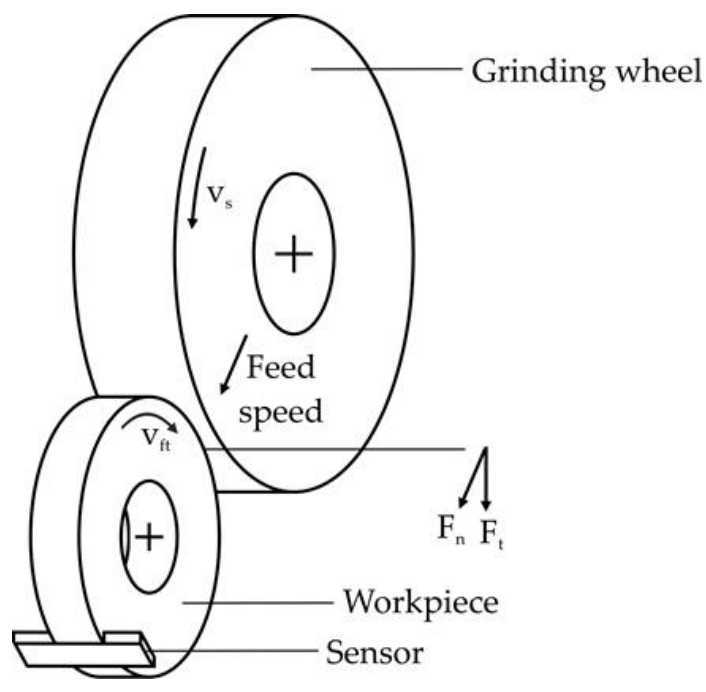

Figure 2. Schematic diagram of the cylindrical grinding process.

a. Development of the sensor inlay by designing a sensor layout and microfabrication of sensors on steel substrates (wafers) using thin-film technology. The sensor inlay is obtained from the fabricated wafers and it consists of a sensor layer with several sensors on it.

b. Embedding the sensor inlay on the actual workpiece that is made of the same steel as the sensor inlay. The workpiece is pre-machined with a cylindrical shape and a groove of $10 \mathrm{~mm} \times 2 \mathrm{~mm}$ is milled on its surface. The sensor inlay is embedded in the groove using epoxy adhesive.

The grinding process in which the sensor-integrated workpiece was used is a cylindrical grinding process. In the process, the rotating grinding wheel plunges into the surface of the workpiece up to a pre-defined depth, the so-called total depth of cut $a_{e}$. After this is reached, the sensor-integrated workpiece starts rotating around its center for one complete revolution so that the full depth of cut $a_{e}$ is removed from the workpiece surface. External forces $F_{\mathrm{t}}$ and $F_{\mathrm{n}}$ during the process induce internal material loads such as strain into the workpiece through its surface. The impact of the internal loads residing in the workpiece leads to material modifications and can be measured from indicators like residual stress. The sensor inlay is positioned on the circumference of the workpiece and, with the help of an underlying sensor layer, measures the load when the grinding wheel moves over it, removing the specific depth of the cut from its surface. This procedure is repeated with a constant depth of cut $a_{e}$ on the same workpiece such that the sensor layer at the bottom of the inlay moves closer to the grinding wheel or the contact zone with every grinding step and finally gets destroyed due to contact with the wheel. The schematic of the procedure is illustrated in Fig. 2. 


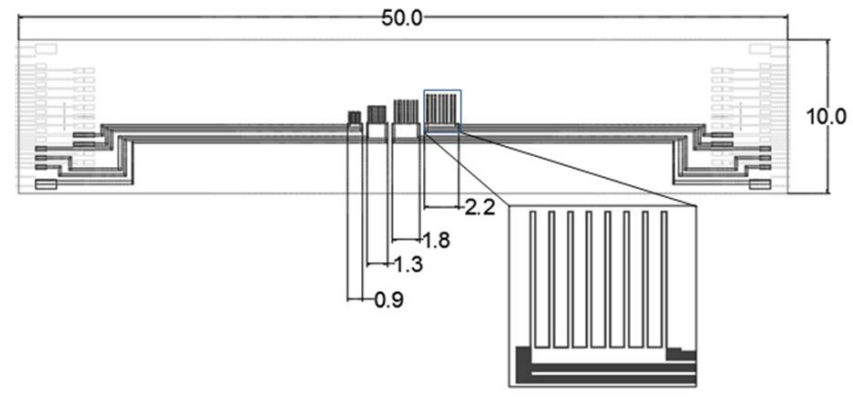

Figure 3. A typical sensor layout, with four resistive structures, on one of the steel inlays. Dimensions are in millimeters.

\section{Materials and methods}

\subsection{Sensor design}

The sensor layer in this work consists of resistive structures in the shape of a meander made of metal films that operates with the same principle as that of a strain gauge. The design of the sensor layer on one sensor inlay has several meanders in various sizes arranged in a specific pattern. A total of 16 sensor inlay layouts were created using the AutoCAD software.

Each individual sensor layout has about four to eight meanders. It should be noted that the strain distribution across the surface of the workpiece during most machining processes is non-uniform, and the microstructure of the material may also introduce variations in the locations of strain fields (Lang and Chou, 1998). This is why meanders of different sizes have been used in the design. Also, more than one meander is used per design in order to facilitate more than one sensor measurement simultaneously during grinding. Each meander is separately connected to a quarter-bridge Wheatstone bridge network externally which consists of the meander as the variable resistor on one arm and three fixed resistors on the other arms, thereby completing the bridge. This has been further explained in Sect. 2.5.

In this work the agglomerated strain over the sensor area is being measured. Hence, in the design most of the meanders are oriented parallel to the cutting direction during machining. The layout is implemented in a photomask used for forming the sensor design on the steel wafers using photolithography. In addition to these 16 designs for the sensor inlays, some designs for test structures were also included. The test structures are square beams with a cross section of $2 \times 2 \mathrm{~mm}$ with resistive meanders. A typical sensor layout design is shown in Fig. 3.

\subsection{Substrate preparation}

The steel wafers for fabricating sensors were cut out in the form of circular discs of $150 \mathrm{~mm}$ in diameter from a sheet of the steel by water-jet cutting. The wafers are very prone to oxidation and for use in microfabrication a long process of lapping, polishing, and smoothing has to be followed. To polish the wafers, chemical-mechanical polishing (CMP) was used. The discs were laminated on a big circular blank and polished on the PM5 polishing machine from Logitech. Both sides of the wafer were lapped gradually with an alumina grit of size $40 \mu \mathrm{m}$ on a lapping disc for about $4-6 \mathrm{~h}$. This brings the wafer to the desired thickness and planarity. After this, the working side of the wafer was polished further, with a suspension of $9 \mu \mathrm{m}$ silicon carbide grit for about $30 \mathrm{~min}$ on the same lapping tool. The surface was then finished with $3 \mu \mathrm{m}$ diamond suspension polishing for 30-60 min. Though this method helped in obtaining polished wafers, there is still the problem of the wafers being non-planar and having a warp of about $300 \mu \mathrm{m}$. This creates a problem in maskalignment lithography. Alternative lithography solutions for non-planar substrates like laser lithography could be useful for the future. After polishing, the wafers were cleaned with acetone, isopropanol, and petrol and taken into the clean room for microfabrication.

\subsection{Material selection}

To select the material of the meanders (sensor layer), it is important that the temperature coefficient of resistance of the material is low and that the gauge factor is high. Also, due to the harsh environment in manufacturing processes, the material should be stable at higher temperatures and higher strain rates. Metal films are the most suitable for this, and here aluminum ( $\mathrm{Al})$ was selected as the sensor material. A major issue in successfully fabricating sensors on robust substrates like steel is the selection of the isolation layer. The sensors have to be well isolated for their proper functioning. But in this case the isolation layer has to provide adequate electrical and chemical insulation to the sensor, not only from the top to protect it from the outside environment, but also from the bottom to avoid connection to the conducting steel substrate. So the sensor element should be sandwiched between two isolation layers. Also, the isolation layer must have good adhesion to steel and should have high thermal and mechanical stability to withstand the continuous mechanical and thermal loading on the sensors when they are used in the machining process (Cheng et al., 2008). From the literature it was found that aluminum oxide $\left(\mathrm{Al}_{2} \mathrm{O}_{3}\right)$ has a thermal expansion co-efficient of $8 \times 10^{-6} \mathrm{~K}^{-1}$, similar to steel, and a high mechanical dielectric strength of $9.9-15.8 \mathrm{kV} \mathrm{mm}^{-1}$ (Choi et al., 2006). It was also found that sputtered $\mathrm{Al}_{2} \mathrm{O}_{3}$ has a very good adhesion to steel (Winkelmann et al., 2011). Due to its good thermal and mechanical properties and good adhesion to steel, it was chosen as the suitable isolation layer.

\subsection{Sensor fabrication}

After polishing and cleaning the wafers, they are taken to a clean room for microfabrication. The first step is to pre- 
pare the surface for deposition of the bottom isolation layer. Though $\mathrm{Al}_{2} \mathrm{O}_{3}$ has good adhesion to steel, a $50 \mathrm{~nm}$ layer of titanium (Ti) was sputtered as an adhesion promoter between the steel and the oxide layer. After this, a sputter etching process at a pressure of $1 \times 10^{-4} \mathrm{~Pa}$ was performed for $30 \mathrm{~s}$ to remove residues from the titanium surface, and then $\mathrm{Al}_{2} \mathrm{O}_{3}$ from a direct target is sputtered for about $2 \mathrm{~h}$ at a pressure of $3 \times 10^{-4} \mathrm{~Pa}$ inside the chamber in an argon gas environment with a flow rate of $106 \mathrm{sccm}$ and a bias voltage of $384 \mathrm{~V}$. This forms the bottom isolation layer and has a total thickness of $200 \mathrm{~nm}$ (Fig. 4a). After this, the sensor material aluminum $(\mathrm{Al})$ is sputtered from a $99.9 \%$ pure $\mathrm{Al}$ target via $\mathrm{DC}$ magnetron sputtering. The total thickness of the $\mathrm{Al}$ layer was $900 \mathrm{~nm}$. The resistive meanders are formed by structuring this layer. Standard photolithography is used for this. A positive resist AZ1518 of $1.8 \mu \mathrm{m}$ thickness was spin coated at a speed of $4500 \mathrm{rpm}$ for $30 \mathrm{~s}$ on the wafer with the LabSpin 6 spin coater from Suss MicroTec that comes with a specialized chuck for holding heavy wafers like the steel wafers and a protective lid to give better working security at higher rpm. A pre-exposure bake of the resist-coated wafer was done in an HTCR oven at $100^{\circ} \mathrm{C}$ for $30 \mathrm{~min}$. The wafers were then aligned with the specific mask and UV exposed in the MA6 mask aligner unit from Suss MicroTec. After developing the exposed resist, the uncovered $\mathrm{Al}$ parts were etched in a phosphoric acid solution at $35^{\circ} \mathrm{C}$ for about $8 \mathrm{~min}$. An etching rate of $140 \mathrm{~nm} \mathrm{~min}^{-1}$ was obtained. With etching, the meanders and their contact pads are formed on the wafer (Fig. 4b).

The meanders have to be covered with a top layer of $\mathrm{Al}_{2} \mathrm{O}_{3}$ and the contact pads have to remain open. For this, the top isolation layer has to be structured, and a lift-off technique was used for this. A negative resist NLOF 2070 of $1.8 \mu \mathrm{m}$ thickness was spin coated on the wafer and developed to form the negative image of the desired pattern of the isolation layer (Fig. 4c). Onto this resist-coated wafer $\mathrm{Al}_{2} \mathrm{O}_{3}$ is deposited. The deposition is done in a three-step process: sputter-evaporation-sputter. A thin layer of $50 \mathrm{~nm}$ was initially sputtered on the wafers. To make the lift-off process easier, the actual thickness of the isolation layer $(280 \mathrm{~nm})$ was obtained by an electron beam evaporation process. The operation temperature of such a process is very low, due to which the underlying resist is protected from overheating or burning during deposition. Also, the directionality as well as line-of-sight geometry of the deposited film in electron beam evaporation makes it very suitable for lift-off (Franssila, 2010). But due to the poor quality of evaporated films, there is always a risk of bigger pinholes on the deposited layer, which is not desirable for isolation layers. To avoid this, a thin layer of $50 \mathrm{~nm}$ of $\mathrm{Al}_{2} \mathrm{O}_{3}$ was sputtered on top of the evaporated film (Fig. 4d).

After depositing the top $\mathrm{Al}_{2} \mathrm{O}_{3}$ layer, the lift-off of the underlying resist was done in a $200 \mathrm{~mL}$ solution of dipropylmonomethyl-ether at $55^{\circ} \mathrm{C}$. The duration of the lift-off process was about $90 \mathrm{~min}$. The steps of fabrication are illustrated

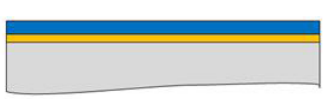

(a)

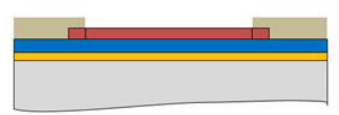

(c)

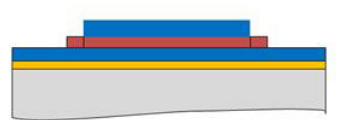

(e)

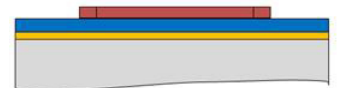

(b)

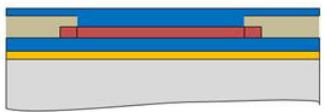

(d)

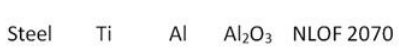

Resist
Figure 4. Steps for fabricating the sensor inlay: (a) sputter deposition of a Ti layer as an adhesion promoter and deposition of the bottom isolation layer, (b) deposition, lithography, and chemical etching of $\mathrm{Al}$ to form the resistive meanders and contact pads, (c) photolithography with negative resist NLOF 2070 for covering the contact pads before deposition of top isolation, (d) deposition of the top isolation layer in a three-step process ( sputtering-evaporationsputtering), and (e) lift-off of the underlying resist to structure the top isolation and open the contact pads for electrical connection.

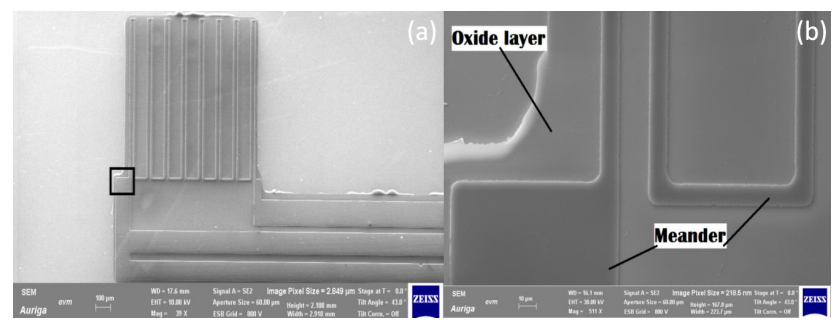

Figure 5. SEM micrographs of (a) one of the fabricated meanders isolated with aluminum oxide from the top; (b) zoomed-out view of the corner of the meander.

in Fig. 4. Microscopic views of the meander and the oxide layer are shown in Fig. 5.

\subsection{Packaging and electronics}

After fabrication, the wafer is diced and sensor inlays as shown in Fig. 6 are obtained from it.

The contact pads of each sensor in the sensor layer are wire bonded to an external circuit board (PCB) using ultrasound and a micro-tip wire bonder. This establishes a contact from the meander to the PCB as shown in Fig. 7. The wire bonds are then coated with epoxy glue and cured for $12 \mathrm{~h}$. This provides mechanical stability. Each meander has three lead wires which are connected to a three-wire quarter bridge configuration of the Wheatstone bridge circuit. The meander is the variable resistor in the circuit and three other resistors (adjustable trim potentiometers) are used externally to complete the Wheatstone network. In unstrained conditions, the 


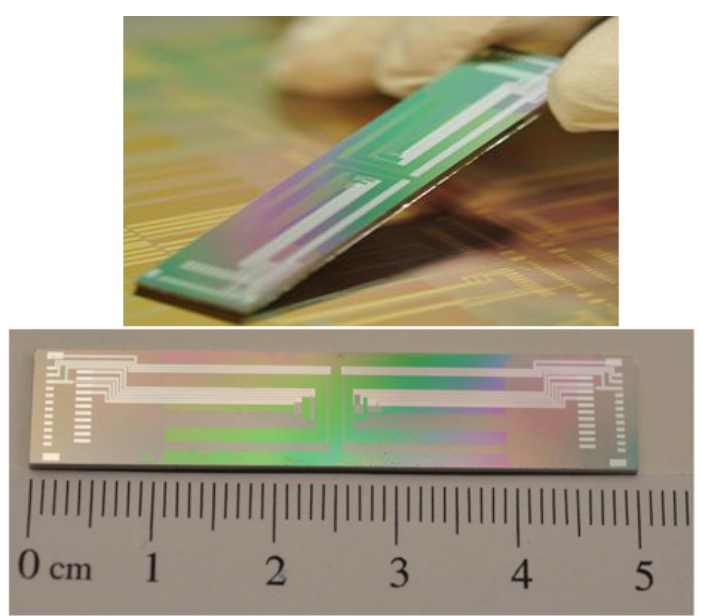

Figure 6. The sensor inlay cut-out from the wafer after fabrication $(50 \times 10 \times 2 \mathrm{~mm})$.

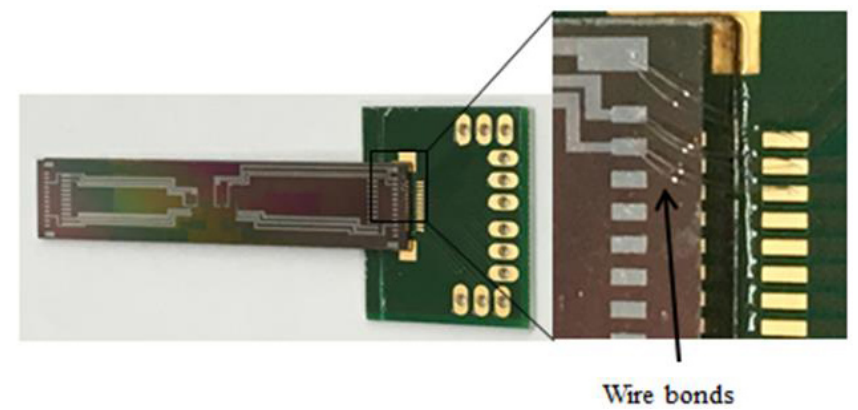

Figure 7. Contact pads of the meander wires bonded to a PCB.

bridge is balanced by adjusting the trim potentiometers so that the output voltage is zero. The circuit is powered with a DC excitation voltage of $5 \mathrm{~V}$ using an external batteryoperated power circuit and the output voltage at each grinding stage is acquired with a NI 6009 data acquisition device (DAQ) from National Instruments. The wire bonded PCB, where the sensor is attached, is covered with a heat-resistant and mechanically stable material.

\subsection{Characterization and calibration}

The strain response and the temperature response of the resistive meanders were calibrated for characterizing the sensors by conducting two separate calibration experiments.

To calibrate the temperature response of the meander, the sensor inlay was placed on a hot plate and its change in resistance to every $5{ }^{\circ} \mathrm{C}$ rise in the temperature was recorded. The sensor inlay was covered with an aluminum box from the top to thermally isolate it from the ambient temperature. A $k$-type thermocouple was mounted directly on the surface of the sensor inlay with the help of a polyimide-based adhesive film to get the true temperature at the surface of the inlay. The initial resistance of the meander at a room temperature

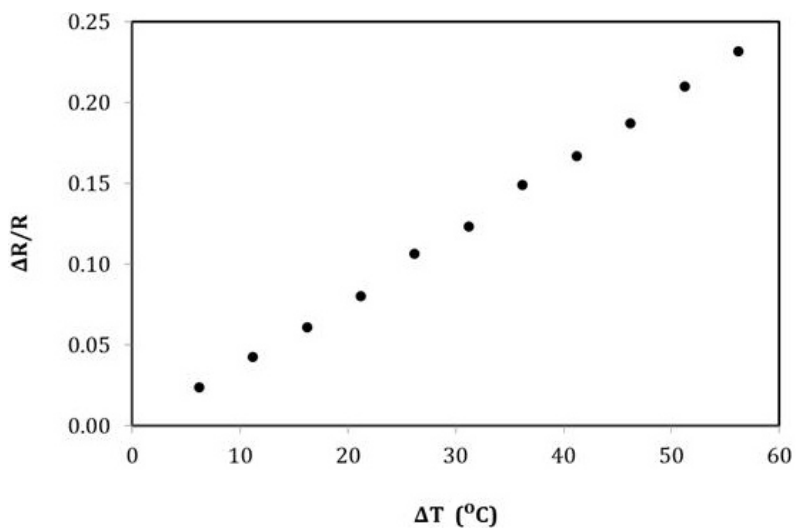

Figure 8. Temperature response showing the change in resistance with respect to change in temperature for a resistive sensor in a sensor inlay.

of $23.8^{\circ} \mathrm{C}$ was found to be $22.46 \Omega$. The change in resistance was recorded with the help of a two-point precision digital multimeter. This is shown as the temperature response curve of the sensor in Fig. 8. From the recorded values of change in resistance, the temperature co-efficient of resistance (TCR) was calculated for 10 thermal loading cycles. The average TCR value was found to be $0.0039^{\circ} \mathrm{C}^{-1}$, which is in accordance with the value found in the literature (Shin and Besser, 2006). The quadratic term of the TCR has been neglected.

For the strain response, the change in resistance due to mechanical strain on the sensor inlay was recorded. A test steel beam $(2 \times 2 \times 10 \mathrm{~mm})$ with a sensor fabricated on it was arranged in a cantilever form with one fixed end and one free end, and a point load was applied at its free end. From the theory of bending of beams, the stress $\sigma$ at a distance of $x$ from the free end of a cantilever beam due to a point load $P$ at the free end is given by

$\sigma=\frac{6 P(l-x)}{b h^{2}}$.

$l, b$, and $h$ are the length, breadth, and height of the beam, respectively. $x$ is the distance between the resistive sensor and the free end of the beam. Hence the strain is measured at this point.

From the stress, the strain $\varepsilon$ due to the applied load can be calculated using Hooke's law:

$\sigma=\varepsilon \cdot E$.

$E$ is the modulus of elasticity of the material of the beam and it is a constant. In this case, the beam is made of $42 \mathrm{CrMo} 4$ steel and the value of $E$ for this particular steel is determined as $2.1 \times 10^{5} \mathrm{MPa}$. The load was applied with a push tool in the Condor 100 machine from XYZTEC. The change in resistance of the sensors due to the applied strain was measured using a precision two-probe digital multimeter. The change in resistance of the fabricated sensors due to applied strain 


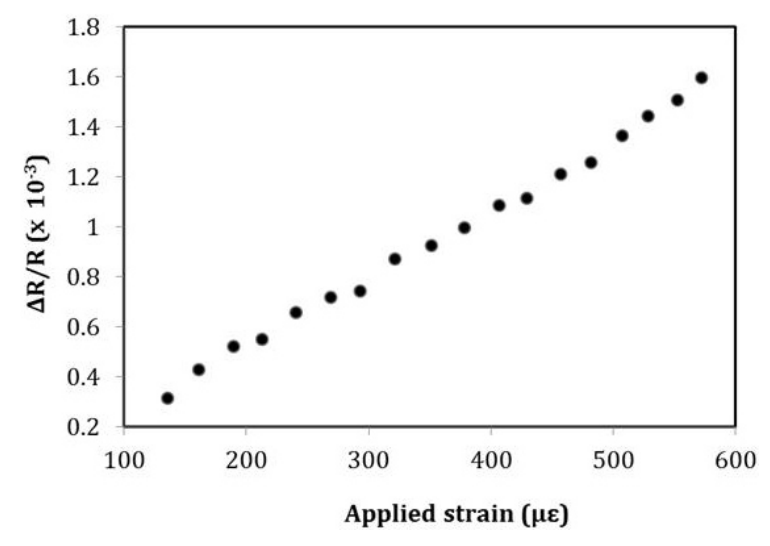

Figure 9. Strain response of the resistive sensors showing the ratio of change in resistance to initial resistance as a result of applied strain.

was measured for 18 progressive loading cycles. This is the strain response curve of the sensor shown in Fig. 9. To calibrate the strain response of the sensor, the sensitivity factor, commonly known as the gauge factor $k$ of the sensor, was measured. From 18 loading cycles, an average gauge factor of 2.64 was calculated.

During experiments, the output voltage of the bridge circuit due to changes in the resistance of the sensor (meander) is acquired as described in Sect. 2.5 at each grinding step over a period of time and later converted to strain. To calculate the measured strain, a standard technique for quarterbridge circuits is used. The strain $(\varepsilon)$ can be derived in terms of the ratio of output voltage $\left(V_{\text {out }}\right)$ to input voltage $\left(V_{\text {in }}\right)$ of a quarter-bridge Wheatstone network, and the gauge factor $(k)$ of the variable resistor or sensor as (Hoffmann, 1989)

$\varepsilon=\frac{-4 \frac{V_{\text {out }}}{V_{\text {in }}}}{k\left(1+2 \frac{V_{\text {out }}}{V_{\text {in }}}\right)}$.

The output voltage $V_{\text {out }}$ is measured after every grinding step, whereas the gauge factor and input voltage $V_{\text {in }}$ remain constant. In this way the strain value is calculated for every grinding step.

The cylindrical grinding process was designed so as to have a maximum temperature gradient of $1-2{ }^{\circ} \mathrm{C}$, which will not have a major effect on the measured strain. A three-wire quarter-bridge circuit was used for additional internal temperature compensation. Also, a constant supply of grinding fluid ensures that the workpiece remains at a constant temperature. Hence, in this work the errors due to temperature have not been taken into account. In the future, it is planned that the meanders will be made of two different materials overlapping each other so that temperature effects can be compensated and the error due to temperature can be quantified.

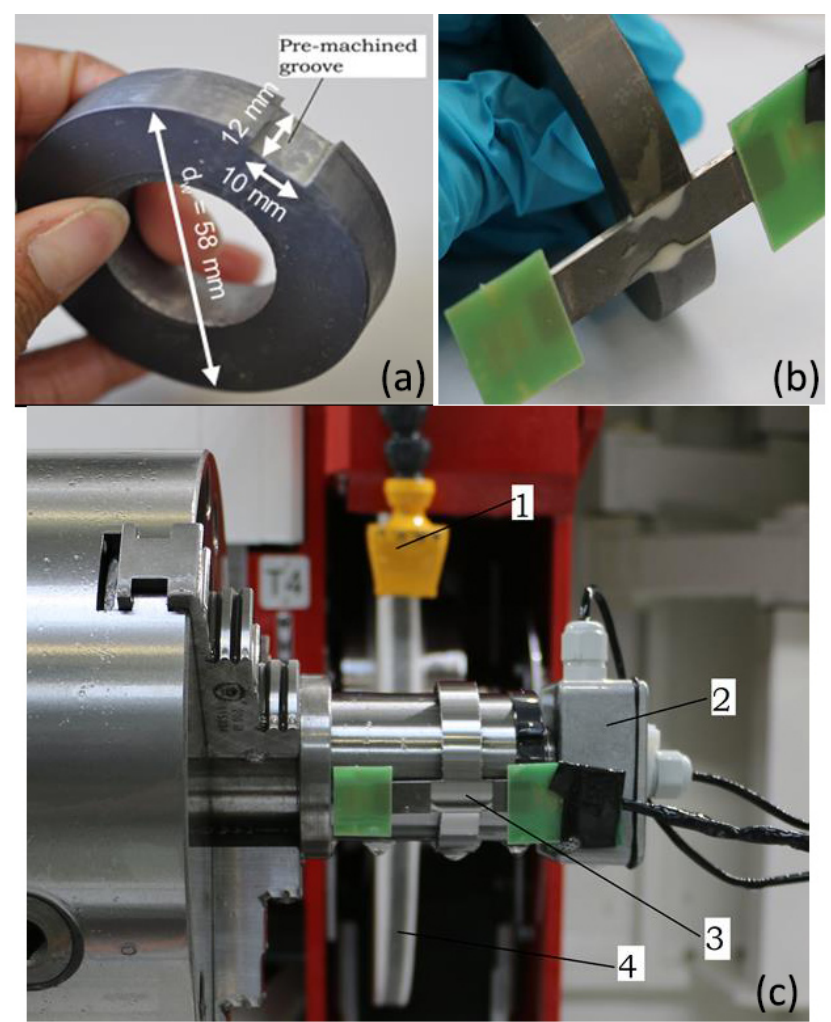

Figure 10. (a) Workpiece with the pre-machined groove to embed the sensor inlay; (b) sensor inlay embedded on the groove upside down; (c) experimental setup: (1) nozzle for coolant, (2) box for holding and protecting the electronic components, (3) sensorintegrated workpiece, and (4) grinding wheel.

\subsection{Embedding the sensor inlay in a workpiece}

For measurement, the fabricated sensor inlays are to be embedded in an AISI4140 steel workpiece. Depending on the manufacturing process, the shape of the workpiece may vary, but the sensor inlay can be embedded in the same way for every suitable workpiece. In this work, the experiments were done in a cylindrical grinding process. Hence, a workpiece, as shown in Fig. 10a, was used and a groove of $12 \times 10 \times 2 \mathrm{~mm}$ was machined on the top surface of the outer diameter of the workpiece, such that the inlay fits into it. The sensor inlay is then turned upside down and fixed in the groove using a two-component epoxy adhesive AremcoBond 526N-ALOX. The groove is made in such a way that only the area covering the sensing element is embedded, while the sensor pads and electronics are hanging freely, as shown in Fig. 10b. The experimental setup is shown in Fig. 10c. The electronics used for acquiring the sensor data were housed in a sealed aluminum box and this was mounted in the machine along with the workpiece in the experimental setup. 
Table 1. Process parameters of the grinding process.

\begin{tabular}{ll}
\hline Material & AISI 4140 (224HV1) \\
\hline Process & Cylindrical grinding \\
Tool & 89A60K5AV \\
Fluid & Low-viscosity grinding oil \\
Grinding wheel speed & $v_{s}=40 \mathrm{~m} \mathrm{~s}^{-1}$ \\
Workpiece tangential & $v_{f t}=0.5 \mathrm{~m} \mathrm{~min}^{-1}$ \\
feed speed & \\
$\begin{array}{l}\text { Depth of cut } \\
\text { Sampling rate for }\end{array}$ & $a_{e}=100 \mu \mathrm{m}$ \\
sensor data acquisition & $100 \mathrm{~Hz}$ \\
\hline
\end{tabular}

\subsection{Experiment}

Two experiments were conducted on a Studer S41 grinding machine which is equipped with an integrated threecomponent force measurement system with piezoelectric sensors mounted on the machine tool. The system is capable of measuring forces ranging from -25 to $25 \mathrm{kN}$. The force sensors are positioned between the workpiece spindle and the machine table. Three force components that can be treated as external loads are thereby obtained: a force in the normal or radial direction to the workpiece $\left(F_{\mathrm{n}}\right)$, a force in the tangential direction of the contact area $\left(F_{\mathrm{t}}\right)$, and the axial force $(\mathrm{Fa})$. In this experiment only a normal feed and a tangential velocity vector were present; hence, only the two components $F_{\mathrm{n}}$ and $F_{\mathrm{t}}$ were evaluated, and the axial force $\mathrm{Fa}$ was neglected. The grinding parameters used are shown in Table 1 . The grinding steps from which sensor data were obtained were repeated on the outer diameter of the top surface of the workpiece. The first step started from $1 \mathrm{~mm}$ above the sensor layer on the sensor inlay, subsequently taking off the so-called "depth of cut" $\left(a_{e}\right)$ of $100 \mu \mathrm{m}$ from the top surface with every step. The sensor data were obtained with a constantly decreasing depth until the sensor inlay was ground down and the sensor layer was destroyed. However, the parameters were constant in every grinding step. The sensor inlay has a flat surface. Hence preliminary grinding steps had to be conducted so that its surface was shaped into a cylindrical form comparable to the rest of the workpiece surface. In this way, one ensured that the grinding wheel was in full contact with the sensor inlay as it moved over the rotating workpiece. The sensor data for these preliminary grinding steps were not evaluated. After a total depth of cut of $1 \mathrm{~mm}$, the surface of the sensor inlay had full contact with the grinding wheel over the entire length of its surface. This was indicated by the external force measurement, displaying a constant load over the contact time of the sensor inlay and the grinding wheel. Measurements were recorded for the subsequent 11 grinding steps. Each grinding step was first executed with a radial feed for plunging until the full depth of cut $a_{e}=100 \mu \mathrm{m}$ was reached.
The sensor inlay is $2 \mathrm{~mm}$ thick. The sensor inlay was positioned at about $180^{\circ}$ of the full rotational movement of the workpiece so that the grooving part of the grinding procedure had a minor influence on the sensor measurement as well as on the run-out at the end of the procedure, at $360^{\circ}$ of the rotation.

The grinding parameters were selected to promote larger mechanical load and low heat. The grinding wheel used has a good cutting ability and generates high surface roughness. It has a relatively coarse grain size and vitrified bond. The grinding wheel is dressed using a dressing procedure. Dressing reduces wear and tear of the wheel which is inevitable due to the abrasive nature of the process and is conducted before each grinding step in the presented work. The dressing procedure was designed to generate a rough grinding wheel surface with increased cutting properties. These measures further ensured a higher mechanical load and a relatively lower thermal load on the sensor inlay and on the workpiece. The grain structure and the surface hardness of the sensor inlay material and the workpiece material, both AISI4140, were made similar by quenching and tempering the workpiece material with the help of a heat treatment process that was specifically designed for this purpose. The resulting surface hardness was 203 HV1 with a standard deviation of $n=7$ for the sensor inlay material and $240 \mathrm{HV} 1$ with a standard deviation of $n=1.6$ for the workpiece material. This relatively small difference in the hardness values ensures that the distribution of the mechanical loads is consistent on the workpiece and on the sensor inlay. To further minimize the heat impact, a high-power metalworking fluid was supplied during the experiments.

\section{Results}

During each grinding step, the strain measured by the sensor layer over the grinding time was recorded. Along with this, the piezoelectric force measurement system of the grinding machine was used to record the tangential and normal forces on the workpiece as external material loads. A strain measurement during the second grinding step over the grinding time, when the tool was $900 \mu \mathrm{m}$ above the sensor layer, is shown in Fig. 11a. The tool touches the workpiece and, as it moves close to the sensor layer, the strain increases and sharply reaches a peak at about $t=8 \mathrm{~s}$. This is due to the maximum strain experienced by the resistive sensor layer as the tool moved directly over it. The total process time for one complete rotation of the workpiece which is calculated from the tangential feed speed and the workpiece diameter was about $21.1 \mathrm{~s}$ at the first grinding step when the workpiece diameter was $56 \mathrm{~mm}$. With every step, the process time decreases due to a constant tangential workpiece speed and a decreasing workpiece diameter. Hence the process time of the evaluated grinding step 2 shown in Fig. $11 \mathrm{~b}$ is about $21 \mathrm{~s}$. The time required for the grinding wheel to move com- 

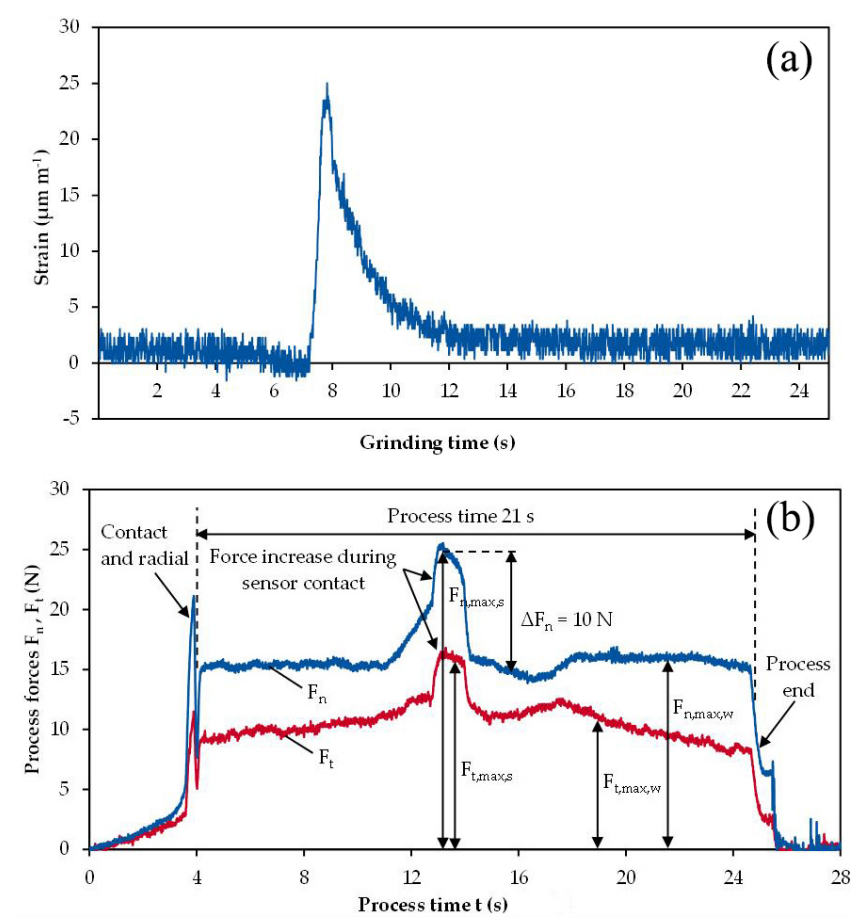

Figure 11. (a) Strain curve as measured by one of the sensors at the second grinding step over time. (b) Process forces in the normal $\left(F_{\mathrm{n}}\right)$ and tangential $\left(F_{\mathrm{t}}\right)$ directions obtained at the second grinding step over the time period of the grinding step.

pletely over the entire length of the sensor inlay $(10 \mathrm{~mm})$ was about $1.2 \mathrm{~s}$. The remaining strain after the grinding wheel has passed over the sensor inlay area of the workpiece is assumed to be caused by the residual heat from the grinding process. Hence the strain does not decrease sharply. The corresponding external force measurement during the fourth grinding step is shown in Fig. 11b. It can be seen that there is a peak at about $t=13 \mathrm{~s}$. This is due to a transition effect caused by the difference in the workpiece and the sensor inlay materials. Another reason for this effect could be the increasing width of cut $a_{p}$ over the sensor inlay area, from $a_{p}=12 \mathrm{~mm}$ over the workpiece material.

The maximum value of the strain recorded during every grinding step can be considered the true strain experienced by the sensor inlay. The maximum strain values at every grinding stage measured simultaneously by two sensors during both experiments are shown in Fig. 12a and b.

The strain induced by the process into the sensor inlay material is actually measured by the change in resistance of the integrated sensors on the workpiece. During grinding, the surface of the workpiece experiences two major forces, normal force and tangential force. To strain the sensors, the resultant force $\left(F_{\mathrm{r}}\right)$ of the normal $\left(F_{\mathrm{n}}\right)$ and tangential force $\left(F_{\mathrm{t}}\right)$ components $\left(F_{\mathrm{r}}^{2}=F_{\mathrm{n}}^{2}+F_{\mathrm{t}}^{2}\right)$ is relevant, which is also the force that causes the process-induced strains in the sensor inlay. The resultants of the maximum measured forces on
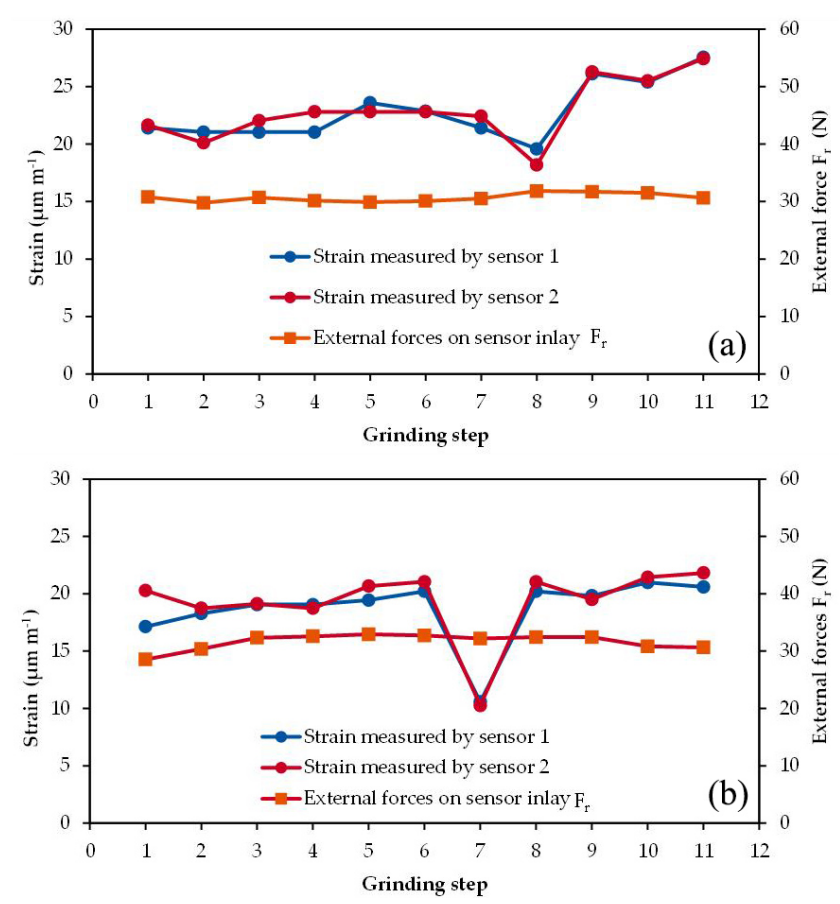

Figure 12. (a) Plot showing the measured process forces and the maximum measured strain at every grinding step during the first experimental series. (b) Plot showing the measured process forces and the maximum measured strain at every grinding step during the second experimental series.

the surface of the sensor inlay, which are denominated as $F_{\mathrm{n}}$, $\max , s$ and $F_{\mathrm{t}}$, max, and $s$ in Fig. $11 \mathrm{~b}$ are plotted in Fig. 12 along with the maximum values of measured strain. It can be deduced that the external forces in each grinding step are approximately equal due to the constant process parameters and similar contact conditions. On the other hand, the internal strain is expected to increase with every step as the tool comes closer and closer to the underlying sensor layer. This is because, as the stage progresses, more material is removed from the top surface and the contact between the tool and the workpiece comes closer to the sensor layer. This is why the internal strain increases after every step even though the external process force remains constant. To prove the validity of the results, besides providing repeated experimental results, preliminary numerical models have been built and the strain curves measured by the sensors could be qualitatively validated, but presenting the models and the results is beyond the scope of this paper.

Comparing both the figures, it can be proved that the sensor measurements are repeatable and consistent because the range of measured strain and the external forces coincide in both sets of experiments. The outlier at grinding step seven in Fig. 12b was probably caused by an error during the experimental procedure and can be neglected. There is a slight increase in the maximum strain measured in the last grinding step when the tool is closest to the sensor layer as compared 
to the measured strain value during the first grinding step, but this increase is not as significant as was expected. A possible reason could be the wound effect of sensor embedding, i.e., the glue surrounding the sensor inlay which could have prevented the sensor inlay from expanding to its full potential under the induced strain. But to prove this, further investigation is required and numerical models have to be built in the future.

\section{Discussion and conclusion}

The results prove that in situ measurement of process quantities like strain is possible with the use of a sensor-integrated workpiece developed as described in this work. A problem arises in fabricating the sensors on the steel wafers due to their non-planarity. In the future, laser lithography will be used as an alternative solution. Also, to separate the effect of temperature from strain, the sensor layer will be fabricated from two different metals and the influence of embedding the sensor inlay in the workpiece by glue will be further investigated.

The goal of the measurements is to record the internal loads during the grinding process and later to generate a transfer function of internal loads. The process forces remain within a narrow range during every grinding step. They alone do not facilitate characterization of the material modifications in the workpiece. This shows that it is not sufficient to measure the external loads alone, but imperative to know the internal material loads and depth profiles to characterize the material modifications and their depth profiles independently of the process as is intended in the so-called process signatures. This can only be done using integrated sensors. With the presented method, it is possible to obtain the internal loads as strain. However, the measurement technique needs to be validated and the results presented are an important contribution to that. Also, the sensor integration to the workpiece needs to be improved for future investigations to obtain the real strain on the workpiece in general and not only on the sensor inlay. So, as mentioned before, the material transition effect should be minimized. The experimental results presented here can be considered to be an important step towards the development of the sensor integration for in situ measurement technology and transferring it to a real machining process. Future work needs to concentrate on complete integration of the sensor in the actual workpiece. Hence, adapting the material properties of the workpiece to make it similar to the sensor inlay material is an important measure to realize a nearly homogenous internal load distribution which the sensor layer can measure. In this way, one can ensure that the sensor layer is able to measure actual strain in the workpiece and not just an equivalent strain on its surface. The measurement of the process forces does not indicate that there is a complete continuum between the workpiece and the sensor inlay due to the increase in forces when the grinding wheel passes the sensor inlay. This should be improvised as it is probably an effect of the change in the contact width $a_{p}$ from the workpiece to the sensor contact as mentioned before. Hence the force and corresponding strain measurements show that the sensor inlay is still decoupled from the workpiece. Another possibility to minimize the transition effect of the grinding wheel from the workpiece to the sensor inlay might be to utilize workpieces with larger diameters so that the unevenness between the flat sensor inlay and a cylindrical workpiece surface is decreased. However, this work is a first step and shows that the sensor principle worked, and first data in a workpiece-integrated sensor inlay during cylindrical grinding of AISI4140 could be repeatedly acquired.

Data availability. Experimental data containing the maximum bridge voltage acquired by the data acquisition system in real time during measurement by both sensors simultaneously, the converted strain values and the maximum external forces (normal and tangential) for both experiments have been made available in Excel files (see Supplement).

\section{The Supplement related to this article is available online at https://doi.org/10.5194/jsss-6-331-2017-supplement.}

Competing interests. The authors declare that they have no conflict of interest.

Acknowledgements. The authors thank the Deutsche Forschungsgemeinschaft (DFG) for funding the present work in subprojects $\mathrm{C} 04$ and $\mathrm{F} 01$ within the transregional Collaborative Research Center SFB/TRR 136.

Edited by: Andreas Schütze

Reviewed by: two anonymous referees

\section{References}

Boedecker, A., Habben, C., Sackmann, A., Budorf, K., Giese, E., and Lang, W.: Manufacturing of a wear detecting sensor made of 17-4PH steel using standard wafer processing technology, Sensor. Actuat. A-Phys., 171, 34-37, 2011.

Brinksmeier, E., Klocke, F., Lucca, D. A., Sölter, J., and Meyer, D.: Process Signatures - A New Approach to Solve the Inverse Surface Integrity Problem in Machining Processes, Procedia CIRP, 13, 429-434, 2014.

Cheng, X., Datta, A., Choi, H., and Li, X.: Metal Embedded Microsensors for Manufacturing Applications, Proceedings of IEEE SENSORS Conference, Daegu, Korea, 22-25 October 2006, https://doi.org/10.1109/ICSENS.2007.355920, 2006. 
Cheng, X. and Li, X.: Development of Metal Embedded Microsensors by Diffusion Bonding and Testing in Milling Process, J. Manuf. Sci. E.-T. ASME, 130, 061010, https://doi.org/10.1115/1.3006318, 2008.

Choi, H., Datta, A., Cheng, X., and Li, X.: Microfabrication and Characterization of Metal-Embedded Thin-Film Thermomechanical Microsensors for Applications in Hostile Manufacturing Environments, J. Microelectromech. S., 15, 322-329, 2006.

Denkena, B., Dahlmann, D., and Boujnah, H.: Sensory workpieces for process monitoring - an approach, Procedia Technology, 26, 129-135, 2016.

Dumstorff, G., Sarma, M., Reimers, M., Kolkwitz, B., Brinksmeier, E., Heinzel, C., and Lang, W.: Steel integrated thin film sensors for characterizing grinding processes, Sensor. Actuat. A-Phys., 242, 203-209, 2016.

Franssila, S.: Introduction to Microfabrication, 2nd Edn., John Wiley \& Sons, Ltd, West Sussex, UK, 289-290, 2010.
Hoffmann, K.: An Introduction to Measurements using Strain Gauges, Hottinger Baldwin Messtechnik GmbH, Darmstadt, Germany, 1989.

Lang, E. J. and Chou, T. W.: The Effect of Strain Gauge Size on Measurement Errors in Textile Component, Elsevier Composites Science \& Technology, 58, 539-548, 1998.

Shin, W. C. and Besser, R. S.: A Micromachined Thin-film Gas Flow Sensor for Microchemical Reactors, J. Micromech. Microeng., 16, 731-741, 2006.

Winkelmann, C., Woitschach, O., Meyer, E. M., and Lang, W.: Development of a strain sensor for rolling contact loads, Proceedings of the 16th International Transducers Conference, Beijing, China, 5-9 June 2011, https://doi.org/10.1109/TRANSDUCERS.2011.5969179, 2011. 